\title{
Correction to: Prediction of Individual Finger Forces Based on Decoded Motoneuron Activities
}

\author{
Chenyun Dai, ${ }^{1}$ Yizhou Cao, ${ }^{1,2}$ and Xiaggang Hu (i) ${ }^{1}$
}

${ }^{1}$ Joint Department of Biomedical Engineering, University of North Carolina at Chapel Hill and North Carolina State University, Chapel Hill, NC 27599, USA; and ${ }^{2}$ Medical College of Soochow University, Suzhou, Jiangsu, China

Correction to: Annals of Biomedical Engineering https://doi.org/10.1007/s10439-019-02240-1

Due to an error in production, Fig. 4 in the original paper shows the root mean squared error (RMSE) between the force and the neural drive estimation. The $R^{2}$ heat map as a function of the number of motor units and the accuracy is illustrated below:

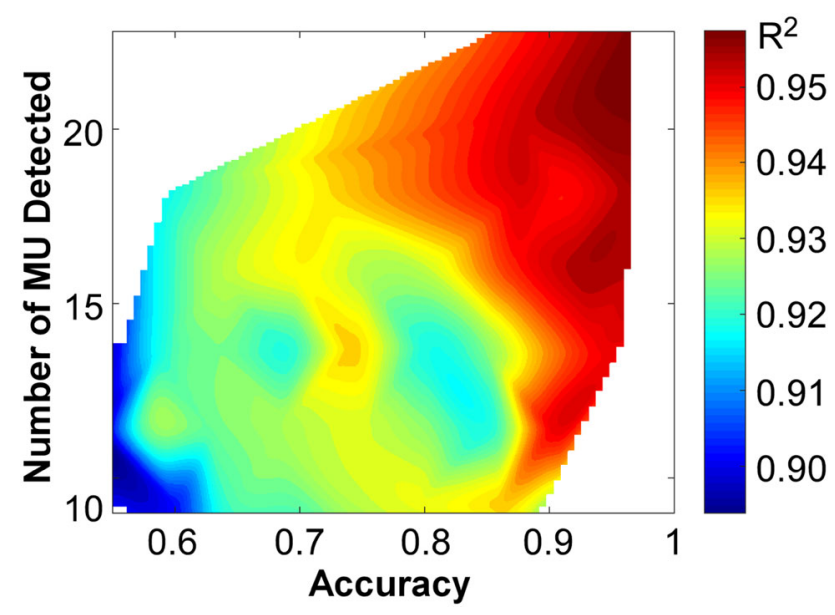

FIGURE 4. The influence of the decomposition performance (accuracy and the number of MUs detected) on the $R^{2}$ of the neural-drive-based estimation. The color bar shows the values of $R^{2}$ s. Note: the map was interpolated linearly three times just for visual presentation.

Publisher's Note Springer Nature remains neutral with regard to jurisdictional claims in published maps and institutional affiliations.

Address correspondence to Xiaogang Hu, Joint Department of Biomedical Engineering, University of North Carolina at Chapel Hill and North Carolina State University, Chapel Hill, NC 27599, USA. Electronic mail: xiaogang@unc.edu

The original article can be found online at https://doi.org/10 1007/s10439-019-02240-1. 